\title{
Effects of grass versus grain on polyunsaturated fatty acids of intramuscular lipids in the lamb
}

\author{
B Aurousseau 1, P Vigneron 2 \\ IINRA, Croissance et Métabolisme des Herbivores, Theix, 63122, St-Genès-Champanelle ; \\ 2INRA, Laboratoire de physiologie animale, 34060 Montpellier cedex, France
}

Comparisons of organoleptic qualities of meat from both cattle and sheep fed on pasture or fed hay and concentrate indoors have lead to contradictory results. The accumulation and derivatization of polyunsaturated fatty acids (PUFA) have been implicated in the differences in aroma of beef obtained from these two types of diets. PUFA were also involved in the occurrence of meat off-flavors, namely in the case of lamb meat. The growing interest of consumers in the quality of their food and the crucial economical difficulties of lamb production require a renewed attention to these topics.

We therefore focused on the relation between lamb management and intramuscular PUFA deposition, in an experiment conducted in the south of France. Two groups of 14 male lambs of the Merinos d'Arles breed were used. The first group was raised outdoors on garrigue (grazing lambs); the second group was weaned at 5 weeks of age and was fed hay and a commercial pellet based on barley (indoor-fed lambs). The lambs were slaughtered over a range of bodyweights from 23 to $36 \mathrm{~kg}$. Triglyceride (TG) and phospholipid (PL) concentrations and fatty-acid composition of three muscles (Supra spinatus ; Longissimus dorsi ; Tensor fasciae latae) were determined as previously described (Bauchart and Aurousseau, 1981, J Dairy Sci, 64, 2033).

As already described in cattle (Miller et al, 1981, J Food Sci, 46, 1333 ; Larick and Turner, 1989, J Anim Sci, 67, 2282), mean (n-3) PUFA contents were significantly higher $(P<0.05)$ in muscle lipids from grazing lambs than in muscle from indoor-fed lambs (2.6 versus $0.9 \%$ for TG, 11.9 versus $6.6 \%$ for $P L$ ) and a reverse tendency was observed for (n-6) PUFA (2.6 versus $3.0 \%$ for TG, 22.4 versus $27.1 \%$, $P<0.05$, for $P L)$. Concentrations of ( $n-6)$ PUFA ( $\mathrm{mg} / \mathrm{g}$ muscle) in PL were similar in muscles from both groups of lambs. The discrepancies between the effects of management on (n-6)
PUFA contents and concentrations in PL appeared partly related to a wide scatter of $\mathrm{PL}$ contents of the muscles (coefficient of variation up to $41 \%$ ) and mainly related to a significantly lower PL content in muscles of indoor-fed lambs $(-3$ to $-21 \%, P<0.05)$. There was a better accordance between contents and concentrations of ( $n-6)$ PUFA in TG and between contents and concentrations of $(n-3)$ PUFA both in TG and PL.

Concentrations of PL and their (n-6) PUFA were highly correlated both in grazing and indoor-fed lambs, but concentrations of PL and their (n-3) PUFA were correlated only in the case of indoor-fed lambs. Concentrations of TG (n-6) PUFA were also highly correlated to total TG, both in the case of grazing and indoor-fed lambs while weaker correlations were obtained between TG (n-3) PUFA and total TG. The rate of increase in PUFA content in $\mathrm{PL}$, along with increase of total PL, was two times higher for $(n-6)$ PUFA than for $(n-3)$ PUFA. The rate of increase in PUFA content in TG was 5 to 6 times higher than in PL in the case of (n-6) PUFA; the difference was even larger in the case of (n-3) PUFA.

In conclusion, PUFA accumulation in intramuscular lipids of the ruminating lamb was significantly influenced by diet; ( $n-3)$ PUFA were increased and (n-6) PUFA were decreased in grazing lambs compared to indoor-fed lambs. The higher scatter of (n-3) PUFA accumulation compared to (n-6) PUFA (both in TG and $P L$ and within the same management group) could be related to greater sensitivity of (n-3) disposal, through individual differences either in hydrogenation in the rumen or in $\beta$-oxidation in the tissues. When the amount of PUFA available for deposition was increased, the well-known efficiency of PUFA sparing in the organism of the ruminant allowed relatively higher changes in the accumulation of PUFA in TG than in $P L$, especially in the case of (n-3) PUFA. 